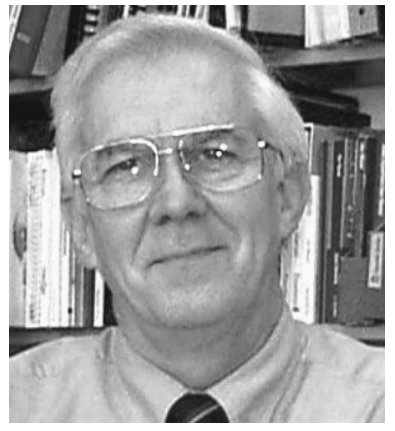

\section{Selling on the Web}

This past Spring quarter I was teamed with my old buddy, Russ Callen, to teach our laser course that is jointly listed by physics and electrical engineering at Georgia Tech. The course was started by Russ, Bill Rhodes and me in the early 1970s and resulted in a textbook by the three of us [Introduction to Lasers and their Applications, Addison-Wesley (1978)].

The teaching responsibility for the course has been shared among a number of our faculty over the years. It had been seven years since I had last taught it. As I reviewed my notes and handouts, I knew I needed to do some updating. Besides, the textbook is a little long in the tooth. So when I was out in San Jose for Photonics West this past January I strolled the aisles of the exhibits, talking to the representatives of a number of laser companies and gathering some product brochures.

Although I am sure that the companies would have mailed me additional brochures to hand out to potential future laser users, by the time I was able to evaluate the material, it would have been a major undertaking to assemble all the materials. So I copied a number of relevant articles on current laser technology to provide my students with information on diode arrays and excimer lasers. (At the time the textbook was written, we had just picked up enough material to be able to describe the earliest versions of excimers and diode lasers were milliwatt sources.)

Then I had a brainstorm. Why not get really up to date? I'll just go to the web! I'll evaluate some laser company web sites and send the URLs to all my students by e-mail. Since class rolls these days include e-mail addresses for the students, it would be a great way to introduce them to new technical resources.

So I launched Netscape and after doing the New York Times crossword, I selected my optics.org bookmark and searched for laser companies. (Disclosure: optics.org is a business venture of SPIE and the Institute of Physics in England.) As I browsed I quickly came to the depressing conclusion that there was not much that I could recommend to my students. Most sites consisted of a few pages that described the major product categories with what I call "box pictures,' pictures of the outside of devices. About the only other content was press releases, which have the educational value equivalent to the writing on bubble gum wrappers.

I did another survey visiting the web sites of those companies that had provided me with a brochure at Photonics West. I wanted to see how the information provided in the brochures correlated with the information on the web. What I found was the smaller the brochure the less correlation between brochure and web site. It might seem that it would be easier to put most of one's brochure on-line if it were short, but it didn't work out that way. It was the heaviest brochure, actually a catalog, that had the most complete web site. My interpretation is that those marketers who value getting information into the customer's hands will do so no matter what the medium.

Still, there was something missing. All of the sites assumed that the browser knew what he or she was looking for. But in this rapidly growing field of optics, that assumption is not tenable. It was a rare site that provided information on the applications of their devices. Those that did so gave only cursory descriptions of no more than 200 words.

During a visit to SPIE headquarters in Bellingham, I talked with some of the staff about the aridity of useful information on lasers. I was told that it wasn't just lasers. Almost all technical web pages are limited in the amount of information they provide. For the most part, the pages give little more than contact information.

Based on what I had found through my directed surfing it appears that the situation wasn't quite as bad as I had first feared. Yet, the information still falls short of what most laser purchasers would need to be able to understand the consequences of a purchase. It is probably too soon to expect the type of content that would be useful to my students, who represent smart, but inexperienced buyers.

I think there are three stages to technical sales on the web: the electronic exhibit, the catalog, and the salesman. Currently most web pages are electronic exhibits. That is, the sites provide a few pictures, some corporate horn tooting, and contact information. They are similar to a real exhibit in that the pages provide more or less what one would get by strolling the aisles of an exhibition and picking up a brochure. Just as in the case of real exhibits, the 
only things that most web sites provide are sales leads.

The sites that do provide catalog information tend to follow their printed versions very closely. One exception that I saw on my browsing tour was a section for fiber communication components. Here a series of schematics of a communications system could be selected until you reached the component level. At that point the various components with their specifications were displayed.

What is missing?

If I give the laser catalogs to my students, they will be able to recognize most of the contents. With the exception of the limited number of applications that we can cover in a ten-week course, there is little they could do with catalogs when faced with a design problem. What is missing is the specific material that would help them understand the trade-offs that are the consequence of their decisions. The sites need to educate ... like a good salesman.

The last stage of technical sales on the web is the salesman or what will represent a salesperson to those seeking information on the web. Obviously high-pressure approaches won't work on the web. After all, you can always walk away by clicking on a new page. But, as the web becomes the prime source for technical information, the tactics that optics suppliers employ will have to change. In my experience as a researcher, the best technical salespeople were those who gave me the information I needed to be able to design my experiments. A good salesperson is part teacher, part hustler. In my estimation, both are missing on the web right now.

One of the most useful aspects of recent optics catalogs has been the inclusion of explanatory material along with product specifications. It provides the purchaser with basic information that he or she can use in assembling a design. Some of this material is specific to the catalog items, other parts are more general and provide the reader with enough understanding to make a considered choice. But there is very little of this in cyberspace.
Obviously there are some aspects of optics and photonics that are common to many products and it would be silly to replicate this material on each web site. It would be useful if a site, such as optics.org, maintained a glossary and a list of short explanations similar to the materials that are available in some of the larger optical component catalogs. Then any site could link to this site for basic explanations and definitions. More specific application materials would be available at the company site. In this way an engineer could be assisted in making a correct purchase.

Once this approach becomes a common sales mode it will be incumbent upon the sellers to provide comparative materials and, perhaps, design materials specific to the task. On one of the sites that I browsed during my survey there was a description of some simulation software for an effect that was produced using the company's lasers. However, the download button was unlinked, so I had no opportunity to evaluate this feature.

Perhaps in the future you might turn to your machine, open a browser to a site and type in: "Need components to recollimate a 633 beam with a $1.3 \mathrm{mrad}$ divergence to one with a 5 microrad divergence"' and get a component list with prices and any discounts. Of course the intelligence engine that drove the output would have to provide counseling, in some cases. "You have just violated the diffraction limit", or "Are you sure? How much money is in your equipment budget?",

The question is: How far could this go? Suppose someone logged on and asked a laser company for a recommendation to cut 1000 board feet of $2 \times 4^{\prime \prime}$ lumber. Would the programmed response be to link to a web page on radial saws at Sears?

Donald C. O'Shea Editor 